\title{
Electrospun PCL-Surgihoney meshes for skin wound healing applications
}

\author{
Enes Aslan ${ }^{1, *}$ \\ ${ }^{1}$ Department of Mechanical, Aerospace and Civil Engineering, University of Manchester, \\ Manchester, UK
}

\begin{abstract}
Skin is a complex and very important tissue, playing a significant protective and regulatory function. It is also prone to a large number of wounds and defects due to external factors such as temperature, chemical agents, and radiation. Accelerating the skin healing process and minimizing the risk of infection is a relevant research challenge. This paper investigates a novel wound dressing based on polycaprolactone (PCL), a synthetic biocompatible and biodegradable polymer, and honeySurgihoney ${ }^{\circledR}(\mathrm{SH})$. Solution electrospinning was used to produce the wound dressing meshes. Different polymer solutions were prepared by mixing PCL and SH with acetic acid. Human dermal fibroblast were used to assess the biological characteristics of the electrospun meshes. Results show that the presence of SH1 has a positive impact on cell attachment and proliferation.
\end{abstract}

\section{Introduction}

Skin is the largest organ in the human body and has a crucial role in protecting the internal organs from the outside environment. When this tissue is disrupted by trauma and disease the natural self-regenerative capacity can be significantly compromised resulting in loss of function and serious health issues. According to the World Health Organisation (WHO), each year worldwide, nearly 300,000 people die as a result of burns and approximately 6 million people are injured [1-3]. Moreover, chronic skin ulcers affect over 6 million people $[2,3]$, which poses a serious economic burden to healthcare systems worldwide and further societal costs due to loss of economic activity. Therefore, of the utmost priority is the development of cost-effective, efficient and effective wound treatment strategies.

Currently, the clinical gold standard to treat critical skin defects is based on the use of skin grafting which uses a healthy tissue taken from the human body (patient or other person) to treat the wounded area [4,5]. However, the use of autografts or allografts both present significant limitations [5]. To overcome the limitations novel wound dressings and artificial tissue engineered skin substitutes are being developed [6,7]. Hover, these dressings are able to protect the wound from the external environment, prevent wound contamination, and dehydration but present limitations in terms of both biological and antibacterial properties.

\footnotetext{
* Corresponding author: enes.aslan@manchester.ac.uk
} 
Electrospinning is a versatile and simple technique to produce nanoscale fibers, highly relevant for the fabrication of wound dressings and meshes for tissue engineering applications $[8,9]$. In the case of tissue engineering, electrospun meshes presents high surface to volume ratios, which enables better attachment of cells. Moreover, the high porosity of such meshes also improves vascularization, oxygen and nutrients exchange [10]. Several studies reported the use of electrospun meshes for a wide range of tissue engineering applications [1,9]. Different synthetic and natural polymers have been investigated $[1,11]$.

Honey has been used to treat wounds since ancient times due to its antiseptic, antibacterial and recuperative properties $[12,13]$. The bactericidal property of honey is due to the production of hydrogen peroxide and reactive oxygen species (ROS), high osmolality of sugar, and low $\mathrm{pH}$ [13-15]. Furthermore, ROS have been demonstrated to promote wound healing by encouraging cellular repair processes and tail regeneration in tadpoles $[16,17]$.

This is paper investigates for the first time the use electrospinning to produce polycaprolactone wound dressings containing different levels of Surgihoney® $(\mathrm{SH})$, a synthetic honey medically approved for skin applications and commercially available in the form of a cream.

\section{Materials and Methods}

\subsection{Materials and Solution Preparation}

Polycaprolactone (PCL) (CAPA 6500, Mw 50,000 g/mol) provided by Perstrop Caprolactones (Cheshire, UK), is a biocompatible polymer used to provide mechanical stability to the meshes. Surgihoney ${ }^{\circledR}(\mathrm{SH})$, provided by Matoke Holding (Abingdom, UK), was used as an antibacterial agent, and comprises different natural honey extracts developed by Matoke. Acetic acid (Fisher Scientific, UK) was selected as a solvent due to its low volatility, non-toxic behaviour and the capacity to dissolve both PCL and honey. Four different PCL-SH $(0 \%, 10 \%, 20 \%$ and $30 \% \mathrm{w} / \mathrm{w}$ of SH) compositions were prepared. Firstly, PCL was dissolved in acetic acid by using a hot plate stirrer overnight at $40^{\circ}$. Then, $\mathrm{SH}$ was added into the mixture and stirred at room temperature until obtaining a homogenous solution.

\subsection{Fabrication}

Electrospinning system can be defined as a cheap and versatile fabrication technique, able to manufacture nanofibrous meshes and highly relevant for the fabrication of wound dressings. Usually, it involves ejecting a polymer solution from a syringe needle tip, by applying a high electric field. A drop of polymer material is formed at the end of the tip due to the effect of surface tension and viscoelastic stresses. The shape of the drop approaches a cone shape (Taylor cone) and a fluid jet is ejected towards the collector.

Electrospun meshes were produced using the Prefector system (Spraybase, Ireland). The electrospinning system consists of a high voltage power supply (from $0 \mathrm{kV}$ to $30 \mathrm{kV}$ ), software to control a syringe pump system, stainless steel collector and a $1.8 \mathrm{Gx} 25 \mathrm{~mm}$ stainless steel emitter (needle). In this case, meshes were produced considering a voltage of $17 \mathrm{kV}$, flow rate of $0.1 \mathrm{~mL} / \mathrm{min}$ and a distance between the needle and the collector of 18.7 $\mathrm{cm}$. 


\subsection{Morphological Analysis}

Morphological characterisation of electrospun meshes was conducted through scanning electron microscopy (SEM, Hitachi S-3000N, Japan) at an accelerating voltage of $15 \mathrm{kV}$. Samples were coated with gold prior to imaging. The images were analysed using Fiji software with the DiameterJ plugin to assess fibre diameter and length.

\subsection{Degradation and Swelling Study}

Degradation and swelling characteristics of produced meshes were investigated using two different media, Dulbecco's Modified Eagle Medium (DMEM) and simulation body fluid (SBF) which was prepared using Kokubo and Takadama's protocol [18]. Samples were cut as $1 \mathrm{~cm} \times 1 \mathrm{~cm}$ square shape and placed into 24 well plates with DMEM and SBF and incubated at $37^{\circ} \mathrm{C}$ during the experiment. Three replicates $(n=3)$ were used to measure the weight as dry and wet. The experiment was conducted for 63 days and the DMEM and SBF were replaced once a week. Same samples were used to measure wet weight $(\mathrm{Ww})$ when conducting swelling study for the first three days, after that different samples $(n=3)$ were used for each time point (days 3, 7, 14, 21, 35, 49 and 63) for the rest of the study (degradation and swelling). Over the course of 9 weeks, samples were removed from the media and the wet electrospun meshes weights $(\mathrm{Ww})$ measured. The swelling ( $\mathrm{Sw})$ was calculated according to the following equation:

$$
S w=\mathrm{W}_{\mathrm{w}} / \mathrm{W}_{\mathrm{i}}
$$

where $\mathrm{Wi}$ is the initial dry weight and $\mathrm{Ww}$ is the weight of the wet electrospun mesh. After, the swollen meshes were dried in the incubator at $37{ }^{\circ} \mathrm{C}$ until completely dry and weighed again (Wd). The percentage of weight loss (Dl) was calculated as follows:

$$
D l=\left(\left(W_{i}-W_{d}\right) / W_{i}\right) * 100
$$

where $\mathrm{W}_{\mathrm{d}}$ is the weight after drying.

\subsection{Biological study}

Electrospun meshes were assessed in terms of cell proliferation using human dermal fibroblast cells (HDF) (Cell Applications,Inc. Sigma Aldrich, UK). Cells were cultured with Dulbecco's Modified Eagle Medium (DMEM) containing 10\% (v/v) fetal bovine serum, $1 \%(\mathrm{v} / \mathrm{v})$ glutamine, and $1 \%(\mathrm{v} / \mathrm{v})$ penicillin/streptomycin until $80 \%$ confluence and harvested using $0.05 \%$ trypsin-EDTA solution (Thermo Fisher Scientific, USA) at passage 2. The metabolic activity of cells, an indicator of cell proliferation, was evaluated using the AlamarBlue ${ }^{\circledR}$ assay kit (Sigma-Aldrich, UK) following the suppliers' protocol. The fluorescence intensity was measured on days 1, 3, 7, and 14 after cell seeding. Four replicates were considered. Cell viability was also assessed using a Live/Dead Assay Kit (ThermoFisher Scientific, UK) at days 3 and 14 according to the manufacturer's instructions.

\section{Results and Discussion}

\subsection{Morphological Analysis}


Figures 1a to 1d present the SEM images and inset fibre distribution graphs of PCL and PCLSH meshes. Results show that the mean fibre diameter decreases with the increase of Surgihoney® concentration (190 nm for PCL; $185 \mathrm{~nm}$ for PCLSH10; $168 \mathrm{~nm}$ for PCLSH20 and $165 \mathrm{~nm}$ for PCLSH30). PCL and PCLSH meshes exhibit a distribution of fiber diameters ranging from $50 \mathrm{~nm}$ to $600 \mathrm{~nm}$. All samples present a uniform fibre distribution. SEM images demonstrate the ability to successfully electrospun both PCL and PCLSH meshes with nanoscale morphology mimicking the native tissue extracellular matrix (ECM).

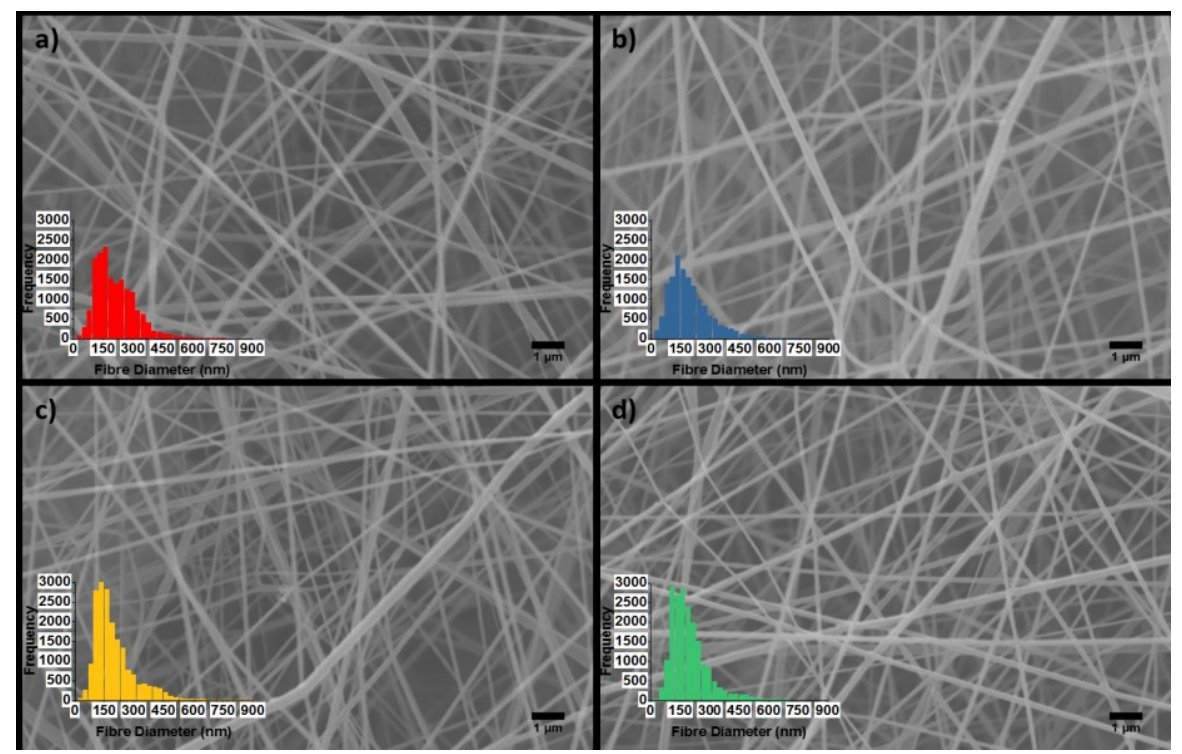

Fig.1. SEM images of electrospun meshes a) PCL, b) PCLSH10, c) PCLSH20, d) PCLSH30 (Scale: $1 \mu \mathrm{m})$. Inset graphs of the distribution of fiber diameters

\subsection{Degradation and Swelling}

The swelling and weight loss values for PCL and PCLSH electrospun meshes are presented in Figure 2. Results show that PCL and PCLSH samples absorb DMEM and SBF until they reach an equilibrium state. It seems that all samples reached out the equilibrium state after day 7 in SBF solution and at day 14 in DMEM. It is also possible to observe that meshes in DMEM absorb more liquid than in SBF. This is due to the fact that meshes start to degrade in SBF solution earlier than in the DMEM. Results also show that meshes containing SH absorb more liquid than PCL meshes. It seems that water molecules containing hydroxyl groups form hydrogen bonds with other hydroxyl groups present in $\mathrm{SH}$ and consequently the liquid absorption is higher in PCLSH meshes than pure PCL meshes. It is also known that ideal wound dressings must be able to absorb the wound exudate. So, the meshes containing SH will be able to absorb more exudate during wound healing and keep the wound bed clean.

Figures $2 \mathrm{c}$ and $2 \mathrm{~d}$ shows the weight loss of the electrospun meshes with time. Results show that PCL meshes present no degradation after day 63 in DMEM, while the meshes lose their half weight in SBF liquid at day 63. On the other hand, the meshes containing SH present a better degradation profile than pure PCL meshes in both liquids. It is also possible to observe that the increase of $\mathrm{SH}$ content on the meshes accelerates the degradation process due to the water solubility of SH. PCLSH30 meshes disappear completely at day 63 in SBF liquid while PCLSH20 meshes loses $86 \%$ of weight. Results also show a faster 
degradation in SBF than DMEM, and, as SBF is able to mimic the body fluids, indicate that PCLSH meshes could be completely degraded in the human body after two months.

a)

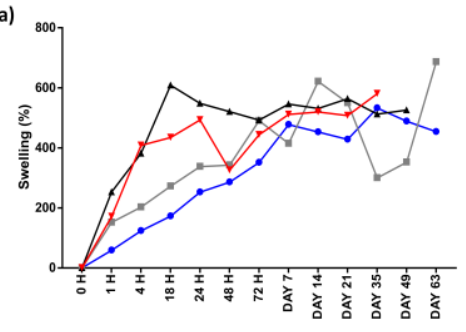

- PCL-SBF - PCLSH10-SBF + PCLSH20-SBF

c)

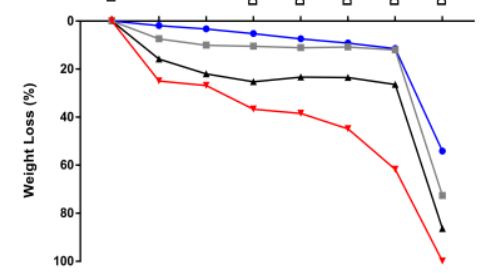

b)

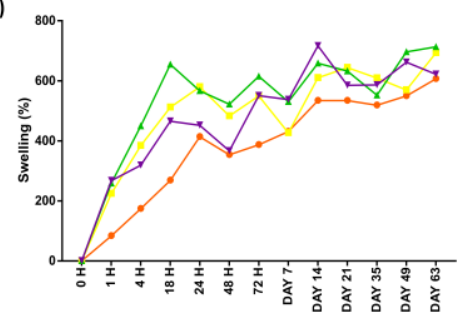

d)

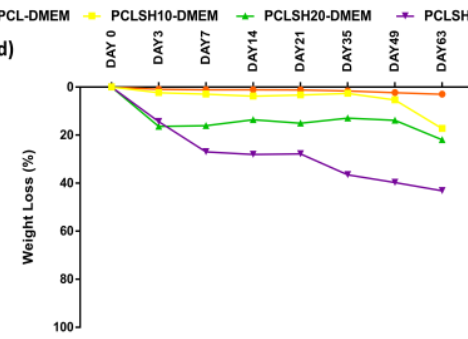

Fig.2. Swelling of the meshes in a) SBF b) DMEM and degradation of the meshes in c) SBF and d) DMEM as a function of time.

\subsection{Biological Results}

Fluorescence intensity values for different time points after cell seeding are presented in Figure 3a. As the fluorescence activity is an indicator of the metabolic activity of cells, results show that all meshes are able to sustain cell attachment and proliferation. Significantly better results are obtained for PCLSH30 meshes at day 14. Figure 3b shows the live cells on the attached meshes and their distribution on the meshes on day 3 and day 14. The results show that cells were able to spread on all samples, creating dense networks, particularly in the case of PCLSH30 meshes containing 30wt $\%$ of SH.

a)

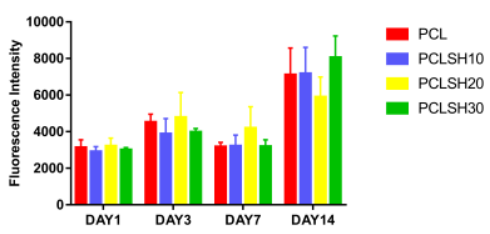

b)
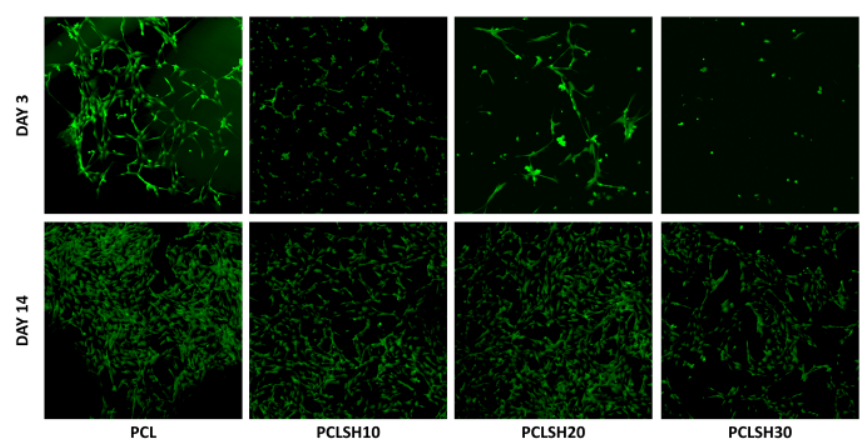

Fig.3. a) Cell proliferation with Alamar Blue Assay at day 1,3,7 and 14 b) Cell viability with Live/Dead staining at day 3 and day 14 . 


\section{Conclusion}

PCL and PCL/SH meshes were successfully produced using solution electrospinning. Meshes present fibers with diameters ranging from 50nm to $600 \mathrm{~nm}$, mimicking the scale of the extracellular matrix (ECM. SH provides hydrophilicity to meshes which leads high water absorption. Meshes containing SH also degrades faster than pure PCL meshes. All meshes supported cell attachment and proliferation with best results obtained for PCLSH30 meshes.

The author wishes to acknowledge the funding provided by the Republic of Turkey Ministry of National Education, and also thanks to his supervisor Prof. Paulo Bartolo for his awesome and supportive guidance. This research was supported by Matoke Holdings.

\section{References}

1. D. R. Figueira, S. P. Miguel, K. D. de Sá, and I. J. Correia, Int. J. Biol. Macromol. 93, $1100(2016)$

2. R. F. Pereira, C. C. Barrias, P. L. Granja, and P. J. Bartolo, Nanomedicine 8, 603 (2013)

3. L. Yildirimer, N. T. K. Thanh, and A. M. Seifalian, Trends Biotechnol. 30, 638 (2012)

4. V. Jayarama Reddy, S. Radhakrishnan, R. Ravichandran, S. Mukherjee, R. Balamurugan, S. Sundarrajan, and S. Ramakrishna, Wound Repair Regen. 21, 1 (2013)

5. F. Groeber, M. Holeiter, M. Hampel, S. Hinderer, and K. Schenke-Layland, Adv. Drug Deliv. Rev. 63, 352 (2011)

6. K. Vig, A. Chaudhari, S. Tripathi, S. Dixit, R. Sahu, S. Pillai, V. A. Dennis, and S. R. Singh, Int. J. Mol. Sci. 18, (2017)

7. R. Augustine, Prog. Biomater. 7, 77 (2018)

8. J. R. Dias, P. L. Granja, and P. J. Bártolo, Prog. Mater. Sci. 84, 314 (2016)

9. P. Zahedi, I. Rezaeian, S. O. Ranaei-Siadat, S. H. Jafari, and P. Supaphol, Polym. Adv. Technol. 21, 77 (2010)

10. C. Vyas, R. Pereira, B. Huang, F. Liu, W. Wang, and P. Bartolo, Curr. Opin. Biomed. Eng. 2, 1 (2017)

11. S. Miguel, M. Ribeiro, P. Coutinho, I. Correia, S. P. Miguel, M. P. Ribeiro, P. Coutinho, and I. J. Correia, Polymers (Basel). 9, 183 (2017)

12. Z. H. Israili, Am. J. Ther. 21, 304 (2014)

13. M. D. Mandal and S. Mandal, Asian Pac. J. Trop. Biomed. 1, 154 (2011)

14. M. Dryden, G. Lockyer, K. Saeed, and J. Cooke, J. Glob. Antimicrob. Resist. 2, 168 (2014)

15. J. Cooke, M. Dryden, T. Patton, J. Brennan, and J. Barrett, BMC Res. Notes 8, 20 (2015)

16. N. R. Love, Y. Chen, S. Ishibashi, P. Kritsiligkou, R. Lea, Y. Koh, J. L. Gallop, K. Dorey, and E. Amaya, Nat. Cell Biol. 15, 222 (2013)

17. C. Dunnill, T. Patton, J. Brennan, J. Barrett, M. Dryden, J. Cooke, D. Leaper, and N. T. Georgopoulos, Int. Wound J. 14, 89 (2017)

18. T. Kokubo and H. Takadama, Biomaterials 27, 2907 (2006) 